\title{
An exact algorithm for constrained $k$-cardinality unbalanced assignment problem
}

\author{
A. Prakash ${ }^{a}$, Uruturu Balakrishna ${ }^{b^{*}}$ and Jayanth Kumar Thenepalle ${ }^{c}$
}

${ }^{a}$ Research Scholar, Department of Mathematics, JNTUA Anantapurammu, India

${ }^{b}$ Professor, Department of Science \& Humanities,Sreeenivasa Institute of Technology and Management Studes, Chittoor, India ${ }^{c}$ Assistant Professor, Department of Science \& Humanities, Sreeenivasa Institute of Technology and Management Studes, India

\section{CH R O N I C L E}

\section{Article history:}

Received May 112021

Received in Revised Format

June 282021

Accepted October 222021

Available online

October, 222021

Keywords:

$k$-cardinality

Unbalanced assignment problem

Zero-one integer programming

Lexi-search algorithm

\section{A B S T R A C T}

An assignment problem (AP) usually deals with how a set of persons/tasks can be assigned to a set of tasks/persons on a one-to-one basis in an optimal manner. It has been observed that balancing among the persons and jobs in several real-world situations is very hard, thus such scenarios can be seen as unbalanced assignment models (UAP) being a lack of workforce. The solution techniques presented in the literature for solving UAP's depend on the assumption to allocate some of the tasks to fictitious persons; those tasks assigned to dummy persons are ignored at the end. However, some situations in which it is inevitable to assign more tasks to a single person. This paper addresses a practical variant of UAP called $k$-cardinality unbalanced assignment problem $(k$ UAP), in which only of persons are asked to perform jobs and all the persons should perform at least one and at most jobs. The $k$-UAP aims to determine the optimal assignment between persons and jobs. To tackle this problem optimally, an enumerative Lexi-search algorithm (LSA) is proposed. A comparative study is carried out to measure the efficiency of the proposed algorithm. The computational results indicate that the suggested LSA is having the great capability of solving the smaller and moderate instances optimally.

\section{Introduction}

The classical assignment problem (AP) is an exceptional case of Transportation problem that aims to seek an optimal schedule between persons $(m)$ and jobs $(n)$ with one-to-one correspondence. Votaw and Orden (1952) however first discussed the concept of assignment problem; the Hungarian method by Kuhn (1955) is the first and significant solution technique for solving AP. Generally, an AP with $m=n$ is known as the balanced assignment problem or simply an assignment problem. However, in most of the practical scenarios, the number of persons and jobs may not be the same (i.e. $m \neq n$ ). A problem of this kind is called unbalanced assignment problem (UAP). Based on the number of persons and jobs involved, the problems can be further classified into two types, namely

(i) UAP with $m<n$

(ii) UAP with $m>n$

Due to the scarcity of workforce, several UAP models can be viewed as problems of the first type (i.e. $m<n)$. These kinds of problems are generally tackled using Hungarian methods and converting unbalanced assignment models into balanced ones by just deploying dummy/ fictitious persons for the first type of problems and dummy/ fictitious jobs for the second type of models. Thus, for the problem of the first kind, few jobs remain idle whereas few persons remain unassigned for the second type of problems. Some of the persons or jobs remain unassigned and thus they persist idle for both kinds of problems. As a result, any organization attains some loss due to not utilizing the resources effectively. Therefore, it is inevitable to address the UAB scenarios in which no persons or jobs are to be left idle.

* Corresponding author

E-mail: prasantibalu@gmail.com (U. Balakrishna)

2022 Growing Science Ltd. doi: $10.5267 /$ j.ijiec.2021.10.002 
Reviewing the literature, Malhotra and Bhatia (1984) addressed two practical variants AP's, where the first one is UAP with $m>n$ and the second one is the UAP with an additional constraint on the minimum number of jobs to be executed by each person. Arora and Puri (1998) studied time minimizing assignment problems in which each job should be performed by precisely a single person and each person can do more than one job with an assumption that all the persons can execute the jobs at the same time. To solve this problem optimally, an exact LSA has been proposed. Kumar (2006) has developed a modified Hungarian method to solve an UAP in which the number of jobs is more than the machines. This modified method effectively assigns the given number of jobs to the machines. Iampang et. al., (2010) considered the UAP with $m>n$ and solves by using a cost and space-efficient methods. This study showed that the solution obtained by cost and space-efficient method is better than that of Kumar's method discussed earlier. Majumdar and Bhunia (2012) presented an efficient Genetic algorithm (GA) for solving UAP with $m<n$. Yadaiah and Haragopal (2016) developed the Lexi-search algorithm for solving UAP with $m>n$. Bhunia et. al., (2017) first considered the UAP with $m<n$ over interval costs together with an additional condition over the number of jobs permitted to do by each person. A GA based solution method is developed to solve this problem effectively. Recently, Thenepalle and Singamsetty (2019) addressed UAP with multiple dimensions and two objectives, where the first objective minimizes the overall time to perform the jobs and the second objective maximizes the overall production on executing the tasks. This model also includes an additional condition over the number of jobs that a person is permitted to perform. To tackle this problem, an exact LSA is developed, which effectively provides efficient Pareto optimal solutions. The works cited above show the significance and scope of UAP with additional practical constraints.

Several variants have been addressed in the literature as AP has practical utility. The $k$-cardinality assignment problem $(k$-AP) is one of the variants of the classical AP, which was first introduced by Dell'Amico and Martello (1997). The so-called $k$-AP can be defined as: Let there are $m$ persons and $n$ jobs, whose cost matrix for performing each job by a person is given by $C=\left(c_{i j}\right)_{m \times n}, c_{i j} \geq 0$. Let a positive integer $k$ where $k \leq m$. The $k$-AP aims to select $k$ persons and $k$ jobs from $m$ persons and $n$ jobs, respectively such that the overall cost incurred is minimized on performing $k$ jobs by $k$ persons. The model $k$-AP has several potential applications namely satellite communication and switching problems (Dell'Amico and Martello, 1997), production planning (Gabrovšek et. al., 2020) etc. With its wide applicability, several researchers have considered $k$-AP and developed various solution methods. For the development of $k$-AP, we may see the works (Dell'Amico and Martello, 1997; Dell'Amico et. al., 2001; Volgenant, 2004; Feng and Yang, 2006; Bai, 2009; Belik and Jörnsten, 2016). However, all the above-cited works on $k$-AP looks for choosing $k$ persons and $k$ jobs from $m$ persons and $n$ jobs, respectively, which still seem a balanced $k$-AP. The models of $k$-cardinality unbalanced assignment problem ( $k$-UAP) with some practical constraints are still open. In addition to the $k$-AP, many researchers have applied the cardinality constraint in various combinatorial optimization problems such as TSP (Bhavani and Murthy, 2006), Minimum spanning tree problem (Kumar and Purusotham, 2017 \& 2018), Multi-objective TSP (Thenepalle and Singamsetty, 2018) etc.

The above-cited works motivate to attempt a practical variant of UAP called $k$-cardinality unbalanced assignment problem with additional constraint ( $k$-UAP). This study also includes a cardinality constraint over the number of persons and an additional condition over number of jobs that is to be done by each person. To deal with this problem optimally, an exact LSA is proposed.

The remaining of the paper is organized as follows: A detailed description of the $k$-UAP and its formulation is given in Section 2. Section 3 describes the preliminaries and the steps involved in the proposed LSA. Section 4 reports computational results. Finally, the conclusions and scope of future work are given in Section 5.

\section{Mathematical Formulation}

Let the sets $I=\{1,2,3, . . m\}$ and $J=\{1,2,3, . . n\}$ consists of $m$ persons and $n$ jobs, respectively with $m<n$. Let $k$ be a non-negative integer such that $k \leq m$. The objective coefficient $c_{i j}$ ( the cost incurred on executing $j^{\text {th }}$ job by $i^{\text {th }}$

person) is defined on each $(i, j) \forall i \in I, j \in J$. Further, an additional condition over $n$ that a person is permitted to execute is also considered. The problem $k$-UAP seeks to find the optimal assignment of performing $n$ jobs by $k$ out of $m$ persons such that each job is to be given to just one person and a person is permitted to execute multiple jobs.

\subsection{Assumptions}

a). Number of persons is less than the number of jobs $(i . e . m<n)$.

b). $n$ jobs are assigned to $k$ out of $m$ persons.

c). Each job is executed by just one person.

d). $m-k$ persons remain unassigned

e). $k$ persons can do at least one and atmost $n-k+1$ jobs.

f). All the jobs are started simultaneously. 
The mathematical model of proposed $k$-UAP using zero-one integer linear programming (0-1 ILP) is as follows:

$$
\begin{aligned}
& \min Z=\sum_{i=1}^{m} \sum_{j=1}^{n} c_{i j} x_{i j} \\
& \text { subject to } \\
& \sum_{i=1}^{m} x_{i j}=1, \forall j \in J \\
& \sum_{j=1}^{n} x_{i j} \geq 0, \forall i \in I \\
& \sum_{i=1}^{m} \sum_{j=1}^{n} x_{i j}=n \\
& 0 \leq \sum_{j=1}^{n} x_{i j} \leq n-k+1, \forall i \in I \\
& x_{i j}=\{1,0\}, \forall i \in I, j \in J
\end{aligned}
$$

Here, total processing cost/time on performing the jobs is minimized through the objective function (1). The Constraint (2) assures that each job should be performed by just one person. The Constraint (3) represents out of $m$ persons, $m-k$ persons remain unassigned whereas the $k$ persons need to do more than or equals to one job. The Constraint (4) ensures that all the given $n$ jobs should be performed. Constraint (5) ensures each of the $k$ persons is allowed to perform at least one and at most $n-k+1$ jobs and remaining persons will not be assigned to any job. Finally, the binary variable $x_{i j}$ in Constraint (6) takes 1 when $i^{\text {th }}$ person performs a $j^{\text {th }}$ job and 0 , otherwise.

\section{Lexi-search Algorithm: Preliminaries}

\subsection{Pattern}

A pattern is usually a two-dimensional matrix $X$ that refers the feasible assignment. The value of the pattern $X$ is computed using the following formula.

$$
V(X)=\sum_{i=1}^{m} \sum_{j=1}^{n} c_{i j} x_{i j}
$$

\subsection{Alphabet table}

The cost matrix $C=\left[c_{i j}\right]_{m \times n}$ consists $m n$ ordered pairs. The elements of $C$ are organized in ascending sequence and marked from 1 to $m n$, saved in an array $S N$ (Sundara Murthy, 1976). If $a_{1}, a_{2} \in S N$ and $a_{1}<a_{2}$ then $C\left(a_{1}\right) \leq C\left(a_{2}\right)$. Let $L_{r}=\left(a_{1}, a_{2}, \ldots, a_{r}\right), a_{i} \in S N$ be a string of $r$ indices. The indices in $L_{r}$ are organized in such a way that $a_{i}<a_{i+1}$ $\forall i=1,2, \ldots, r-1$. The word $L_{r}$ with $a_{i}<a_{i+1} \forall i=1,2, \ldots, r-1$ is termed as 'sensible word' and otherwise, it is referred to as 'non-sensible word'. The systematic sequence of $r$ indices from $S N$ is known as a 'word' of length $r$. The word $L_{r}$ of length $r<n$ is said to be the partial feasible word, whereas the word $L_{r}$ having length $r=n$ is called a complete feasible word.

\subsection{Lexi-search mechanism}

The Lexi-search algorithm (LSA) is a systematized Branch \& Bound (B\&B) approach, proposed by Pandit (1962) for resolving the loading problem. This approach is proved to have a great capability in solving many combinatorial optimization problems optimally (Pandit,1962).

"It is possible to list out all the solutions in a systematic hierarchy, which also have a hierarchical ordering of the corresponding values of the solutions". 
The performance of the LSA usually be governed by on the choice of the suitable alphabet table, which can be done in two ways (Sundara Murthy, 1976).

"The process of checking the feasibility of a partial word is easy; on the other hand, the calculation of lower bound is complex".

"The computation of lower bound is easy; while the feasibility checking is difficult".

\subsection{Bounds Calculation}

As the objective function $Z$ is of minimization type, the upper bound (TS=UB) of $Z$ is supposed to have a very large value. The lower bound $L B$ and the value of $V$ for a partial word $L_{r}$ is determined as follows:

$L B\left(L_{r}\right)=V\left(L_{r}\right)+C T\left(a_{r}+n-r\right)-C T\left(a_{r}\right)$; where $V\left(L_{r}\right)=V\left(L_{r-1}\right)+C\left(\mathrm{a}_{r}\right)$ with $V\left(L_{0}\right)=0$

\subsection{Proposed LSA}

The step by step process of proposed LSA for $k-U A P$ is illustrated below.

Step-1: Initialization

$k$ and cost matrix $\left(C=\left[c_{i j}\right]\right)$.

Set $T S=U B=9999$.

Step-2: If the problem dimension fulfills the condition $m<n$ and $k \leq m$, then move to Step 3; else, no feasible solution for the problem and move to Step- 14 .

Step-3: Generate the alphabet table for the cost matrix $(C)$. Move to Step-4.

Step-4: The search begins with $L_{r}=\left(a_{1}\right)=1$. Move to Step-5.

Step-5: Calculate $L B\left(L_{r}\right)$. If $L B\left(L_{r}\right)<T S$ then go to Step-6; else, move to Step-10.

Step-6: If $L_{r}$ is feasible, then we admit it and remain for next partial word of order $r+1$ and go to Step-7; else, consider the successive partial word of order $r$ by taking another letter that succeeds $a_{r}$ in its $r^{\text {th }}$ position and move to Step-5.

Step-7: If $r=n$ then go to Step-8; else, go to Step-9.

Step-8: Update $T S$ by $L B\left(L_{r}\right)$ and move to Step-11.

Step-9: The partial word $L_{r}$ can be concatenated by using $L_{r}=L_{r-1} \otimes\left(a_{r}\right)$ where $\otimes$ defines the concatenation operation and go to Step-5.

Step-10: If $r=1$, then go to Step-14; else move to Step-11.

Step-11: Take $T S=U B$ and proceed further by taking $r=r-1$ and go to Step-5.

Step-12: Continue the Steps 5-11 sequentially till TS has no further development and go to Step-13.

Step-13: Record TS . Move to Step-14.

Step-14: Stop

Finally, the currrent $T S$ gives the optimal solution.

\section{Numerical Example}

To demonstrate the proposed LSA, a suitable example with five persons $(m=5)$, seven jobs $(n=7)$ and cardinality over number of persons is restricted to two $(k=2)$ is considered. The person-job assignment matrix $C=\left[c_{i j}\right]$ is provided in Table 1 .

Table 1

The cost of performing every job by each person

\begin{tabular}{|c|c|c|c|c|c|c|c|}
\hline & $J_{I}$ & $J_{2}$ & $J_{3}$ & $J_{4}$ & $J_{5}$ & $J_{6}$ & $J_{7}$ \\
\hline$P_{1}$ & 21 & 12 & 2 & 26 & 21 & 7 & 18 \\
\hline$P_{2}$ & 9 & 10 & 5 & 22 & 25 & 12 & 20 \\
\hline$P_{3}$ & 15 & 24 & 30 & 16 & 29 & 21 & 15 \\
\hline$P_{4}$ & 19 & 28 & 11 & 32 & 27 & 17 & 26 \\
\hline$P_{5}$ & 14 & 23 & 26 & 2 & 3 & 4 & 3 \\
\hline
\end{tabular}


The alphabet table for the cost matrix $C=\left[c_{i j}\right]$ is provided in Table 2. In Table 2, the the array notations $S N, C t, C C t, R, C$ indicates Index number, values of cost matrix in ascending order, cumulative cost, row, column indices, respectively.

Table 2

Alphabet table

\begin{tabular}{|c|c|c|c|c|c|c|c|c|c|}
\hline$S N$ & $C t$ & $C C t$ & $R$ & $C$ & $S N$ & $C t$ & $C C t$ & $R$ & $C$ \\
\hline 1 & 2 & 2 & 1 & 3 & 19 & 19 & 194 & 4 & 1 \\
\hline 2 & 2 & 4 & 5 & 4 & 20 & 20 & 214 & 2 & 7 \\
\hline 3 & 3 & 7 & 5 & 5 & 21 & 21 & 235 & 1 & 1 \\
\hline 4 & 3 & 10 & 5 & 7 & 22 & 21 & 256 & 1 & 5 \\
\hline 5 & 4 & 14 & 5 & 6 & 23 & 21 & 277 & 3 & 6 \\
\hline 6 & 5 & 19 & 2 & 3 & 24 & 22 & 299 & 2 & 4 \\
\hline 7 & 7 & 26 & 1 & 6 & 25 & 23 & 322 & 5 & 2 \\
\hline 8 & 9 & 35 & 2 & 1 & 26 & 24 & 346 & 3 & 2 \\
\hline 9 & 10 & 45 & 2 & 2 & 27 & 25 & 371 & 2 & 5 \\
\hline 10 & 11 & 56 & 4 & 3 & 28 & 26 & 397 & 1 & 4 \\
\hline 11 & 12 & 68 & 1 & 2 & 29 & 26 & 423 & 4 & 7 \\
\hline 12 & 12 & 80 & 2 & 6 & 30 & 26 & 449 & 5 & 3 \\
\hline 13 & 14 & 94 & 5 & 1 & 31 & 27 & 476 & 4 & 5 \\
\hline 14 & 15 & 109 & 3 & 1 & 32 & 28 & 504 & 4 & 2 \\
\hline 15 & 15 & 124 & 3 & 7 & 33 & 29 & 533 & 3 & 5 \\
\hline 16 & 16 & 140 & 3 & 4 & 34 & 30 & 563 & 3 & 3 \\
\hline 17 & 17 & 157 & 4 & 6 & 35 & 32 & 595 & 4 & 4 \\
\hline 18 & 18 & 175 & 1 & 7 & & & & & \\
\hline
\end{tabular}

The logical flow of the proposed LSA is presented in Table 3. The column $S N$ in Table 3 denotes the index number. As the total jobs done by two persons is seven, thus the length of the word $L_{r}$ becomes 7 . Hence, the columns after $S N$ are denoted by $1,2,3,4,5,6,7$, each one indicating the letters in corresponding places of a word. The next two columns $V c \& L B$ represent the value and lower bound of a partial word $L_{r}$, respectively. The subsequent two columns $R$ and $C$, respectively denotes the row and column indices of the respective letter. Finally, the last column Remark represents accept the partial word when it is feasible and marked as $A c$ i.e Accept; otherwise $R_{j}$ i.e reject. Initially, the algorithm starts by assuming a trial solution $T S=999999$, a large value. For each $S N, L B$ of a partial word is evaluated and confirmed if it fulfills the bounds or not. A partial word is marked $A c$ only when the $L B$ is less than the trial solution and satisfies the constraints given in Section 3. A partial word is marked $R j$ when it fails to satisfy any one of the constraints given in Section 3 . Similarly, if the $L B \geq T S$, then it is rejected and denoted by $\geq T S$, Rj. In Table 3 , it is seen that the initial feasible solution $(T S=40)$ is found at $13^{\text {th }}$ row and the respective feasible word is $L_{7}=(1,2,3,4,5,11,13)$.

Table 3

Logical flow of proposed LSA

\begin{tabular}{|c|c|c|c|c|c|c|c|c|c|c|c|c|}
\hline$S N$ & 1 & 2 & 3 & 4 & 5 & 6 & 7 & $V$ & $L B$ & $R$ & $C$ & Remark \\
\hline 1 & 1 & & & & & & & 2 & 26 & 1 & 3 & $A c$ \\
\hline 2 & & 2 & & & & & & 4 & 26 & 5 & 4 & $A c$ \\
\hline 3 & & & 3 & & & & & 7 & 26 & 5 & 5 & $A c$ \\
\hline 4 & & & & 4 & & & & 10 & 26 & 5 & 7 & $A c$ \\
\hline 5 & & & & & 5 & & & 14 & 26 & 5 & 6 & $A c$ \\
\hline 6 & & & & & & 6 & & 19 & 26 & 2 & 3 & $R j$ \\
\hline 7 & & & & & & 7 & & 21 & 30 & 1 & 6 & $R j$ \\
\hline 8 & & & & & & 8 & & 23 & 33 & 2 & 1 & $R j$ \\
\hline 9 & & & & & & 9 & & 24 & 35 & 2 & 2 & $R j$ \\
\hline 10 & & & & & & 10 & & 25 & 37 & 4 & 3 & $R j$ \\
\hline 11 & & & & & & 11 & & 26 & 38 & 1 & 2 & $A c$ \\
\hline 12 & & & & & & & 12 & 38 & 38 & 2 & 6 & $R j$ \\
\hline 13 & & & & & & & 13 & 40 & 40 & 5 & 1 & $A, T S=40$ \\
\hline 14 & & & & & & 12 & & 26 & 40 & 2 & 6 & $\geq T S, R j$ \\
\hline 15 & & & & & 6 & & & 15 & 31 & 2 & 3 & $R j$ \\
\hline 16 & & & & & 7 & & & 17 & 36 & 1 & 6 & $A c$ \\
\hline 17 & & & & & & 8 & & 26 & 36 & 2 & 1 & $R j$ \\
\hline 18 & & & & & & 9 & & 27 & 38 & 2 & 2 & $R j$ \\
\hline 19 & & & & & & 10 & & 28 & 40 & 4 & 3 & $\geq T S, R j$ \\
\hline 20 & & & & & 8 & & & 19 & 40 & 2 & 1 & $\geq T S, R j$ \\
\hline 21 & & & & 5 & & & & 11 & 32 & 5 & 6 & $A c$ \\
\hline 22 & & & & & 6 & & & 16 & 32 & 2 & 3 & $R j$ \\
\hline 23 & & & & & 7 & & & 18 & 37 & 1 & 6 & $R j$ \\
\hline 24 & & & & & 8 & & & 20 & 41 & 2 & 1 & $\geq T S, R j$ \\
\hline 25 & & & & 6 & & & & 12 & 38 & 2 & 3 & $\overline{R j}$ \\
\hline
\end{tabular}


Table 3

Logical flow of proposed LSA (Continued)

\begin{tabular}{|c|c|c|c|c|c|c|c|c|c|c|c|c|}
\hline$S N$ & 1 & 2 & 3 & 4 & 5 & 6 & 7 & $V$ & $L B$ & $R$ & $C$ & Remark \\
\hline 26 & & & & 7 & & & & 14 & 44 & 1 & 6 & $\geq T S, R j$ \\
\hline 27 & & & 4 & & & & & 7 & 32 & 5 & 7 & $A c$ \\
\hline 28 & & & & 5 & & & & 11 & 32 & 5 & 6 & $A c$ \\
\hline 29 & & & & & 6 & & & 16 & 32 & 2 & 3 & $R j$ \\
\hline 30 & & & & & 7 & & & 18 & 37 & 1 & 6 & $R j$ \\
\hline 31 & & & & & 8 & & & 20 & 41 & 2 & 1 & $\geq T S, R j$ \\
\hline 32 & & & & 6 & & & & 12 & 38 & 2 & 3 & $R j$ \\
\hline 33 & & & & 7 & & & & 14 & 44 & 1 & 6 & $\geq T S, R j$ \\
\hline 34 & & & 5 & & & & & 8 & 39 & 5 & 6 & $A c$ \\
\hline 35 & & & & 6 & & & & 13 & 39 & 2 & 3 & $R j$ \\
\hline 36 & & & & 7 & & & & 15 & 45 & 1 & 6 & $\geq T S, R j$ \\
\hline 37 & & & 6 & & & & & 9 & 46 & 2 & 3 & $\geq T S, R j$ \\
\hline 38 & & 3 & & & & & & 5 & 33 & 5 & 5 & $A c$ \\
\hline 39 & & & 4 & & & & & 8 & 33 & 5 & 7 & $A c$ \\
\hline 40 & & & & 5 & & & & 12 & 33 & 5 & 6 & $A c$ \\
\hline 41 & & & & & 6 & & & 17 & 33 & 2 & 3 & $R j$ \\
\hline 42 & & & & & 7 & & & 19 & 38 & 1 & 6 & $R j$ \\
\hline 43 & & & & & 8 & & & 21 & 42 & 2 & 1 & $\geq T S, R j$ \\
\hline 44 & & & & 6 & & & & 13 & 39 & 2 & 3 & $\overline{R j}$ \\
\hline 45 & & & & 7 & & & & 15 & 45 & 1 & 6 & $\geq T S, R j$ \\
\hline 46 & & & 5 & & & & & 9 & 40 & 5 & 6 & $\geq T S, R j$ \\
\hline 47 & & 4 & & & & & & 5 & 40 & 5 & 7 & $\geq T S, R j$ \\
\hline 48 & 2 & & & & & & & 2 & 33 & 5 & 4 & $\overline{A c}$ \\
\hline 49 & & 3 & & & & & & 5 & 33 & 5 & 5 & $A c$ \\
\hline 50 & & & 4 & & & & & 8 & 33 & 5 & 7 & $A c$ \\
\hline 51 & & & & 5 & & & & 12 & 33 & 5 & 6 & $A c$ \\
\hline 52 & & & & & 6 & & & 17 & 33 & 2 & 3 & $A c$ \\
\hline 53 & & & & & & 7 & & 24 & 33 & 1 & 6 & $R j$ \\
\hline 54 & & & & & & 8 & & 26 & 36 & 2 & 1 & $A c$ \\
\hline 55 & & & & & & & 9 & 26 & 36 & 2 & 2 & $A c, T S=36$ \\
\hline 56 & & & & & & 9 & & 27 & 37 & 2 & 2 & $\geq T S, R j$ \\
\hline 57 & & & & & 7 & & & 19 & 38 & 1 & 6 & $\geq T S, R j$ \\
\hline 58 & & & & 6 & & & & 13 & 39 & 2 & 3 & $\geq T S, R j$ \\
\hline 59 & & & 5 & & & & & 9 & 40 & 5 & 6 & $\geq T S, R j$ \\
\hline 60 & & 4 & & & & & & 5 & 40 & 5 & 7 & $\geq T S, R j$ \\
\hline 61 & 3 & & & & & & & 3 & 41 & 5 & 5 & $\geq T S, R j$ \\
\hline
\end{tabular}

To improve the solution, a backtracking strategy is applied and the next improved solution $(T S=36)$ is obtained at $55^{\text {th }}$ row of the Table 3, whose feasible word is $L_{7}=(2,3,4,5,8,9)$. Since there is no further improvement of the current solution $(V T=36)$, the search process completes at $61^{\text {st }}$ row and the latest found solution $(V T=36)$ is considered as the optimal solution. The fulllength words of feasible and optimal solutions and their corresponding schedules are reported in Table 4.

Table 4

Feasible and optimal schedules

\begin{tabular}{llll}
\hline SN & Full-length word & Feasible/Optimal Schedule & Feasible/ Optimal Solution \\
\hline 1 & $\mathrm{~L}_{7}=(1,2,3,4,5,11,13)$ & $(1,3),(5,4),(5,5),(5,7),(5,6),(1,2),(5,1)$ & 40 \\
2 & $\mathrm{~L}_{7}=(2,3,4,5,6,8,9)$ & $(5,4),(5,5),(5,7),(5,6),(2,3),(2,1),(2,2)$ & 36 \\
\hline
\end{tabular}

The feasible and optimal schedules reported in Table 4 are also represented as $P_{1} \rightarrow J_{2} \wedge J_{3}, P_{5} \rightarrow J_{1} \wedge J_{4} \wedge J_{5} \wedge J_{6} \wedge J_{7}$ and $P_{2} \rightarrow J_{1} \wedge J_{2} \wedge J_{3}, P_{5} \rightarrow J_{4} \wedge J_{5} \wedge J_{6} \wedge J_{7}$ respectively. For better understanding $P_{1} \rightarrow J_{2} \wedge J_{3}$ indicates that second and third jobs are performed by first person. Similarly, $P_{5} \rightarrow J_{4} \wedge J_{5} \wedge J_{6} \wedge J_{7}$ denotes fourth, fifth, sixth and seventh jobs are done by fifth person.

\section{Computational Results}

All the experiments were tested in Matlab 2017a, Intel Core i5 2.10 with GHz CPU B950 and 4 GB of RAM PC running Microsoft Windows 2010 OS. The computational results including comparative results of relaxed version of $k$ - $U A P$ and computational results of $k$-UAP using proposed LSA tested on some selected test instances as used by Majumdar \& Bhunia (2012). To compare the results of the proposed LSA against the results of Majumdar \& Bhunia (2012), the current model $k$ $U A P$ will transform to an equivalent model addressed by Majumdar \& Bhunia (2012) by setting $k=m$ and imposing a constraint over the jobs that are permitted to do by each person.

\subsection{Comparision of proposed LSA and Genetic Algorithm of Majumdar \& Bhunia (2012)}

The proposed LSA performance can be assessed through a comparative study of the genetic algorithm (GA) developed by Majumdar and Bhunia (2012) for UAP with $m<n$, whose best GA results (GA*) are considered for comparison purposes. These results are acquired by testing two distinct variants of GA (GA-1 and GA-2) over the cases of sizes $5 \times 7,5 \times 8,6 \times 10$ 
and $7 \times 10$ with distinct parametric values. Note that these instances are denoted as AP-1, AP-2, AP-3 and AP-4, respectively. All these instances are also provided in the Appendix. Tables 5-7 provide the comparative details of LSA and GA*

Table 5

Comparative results of LSA against GA* for AP-1 and AP-2

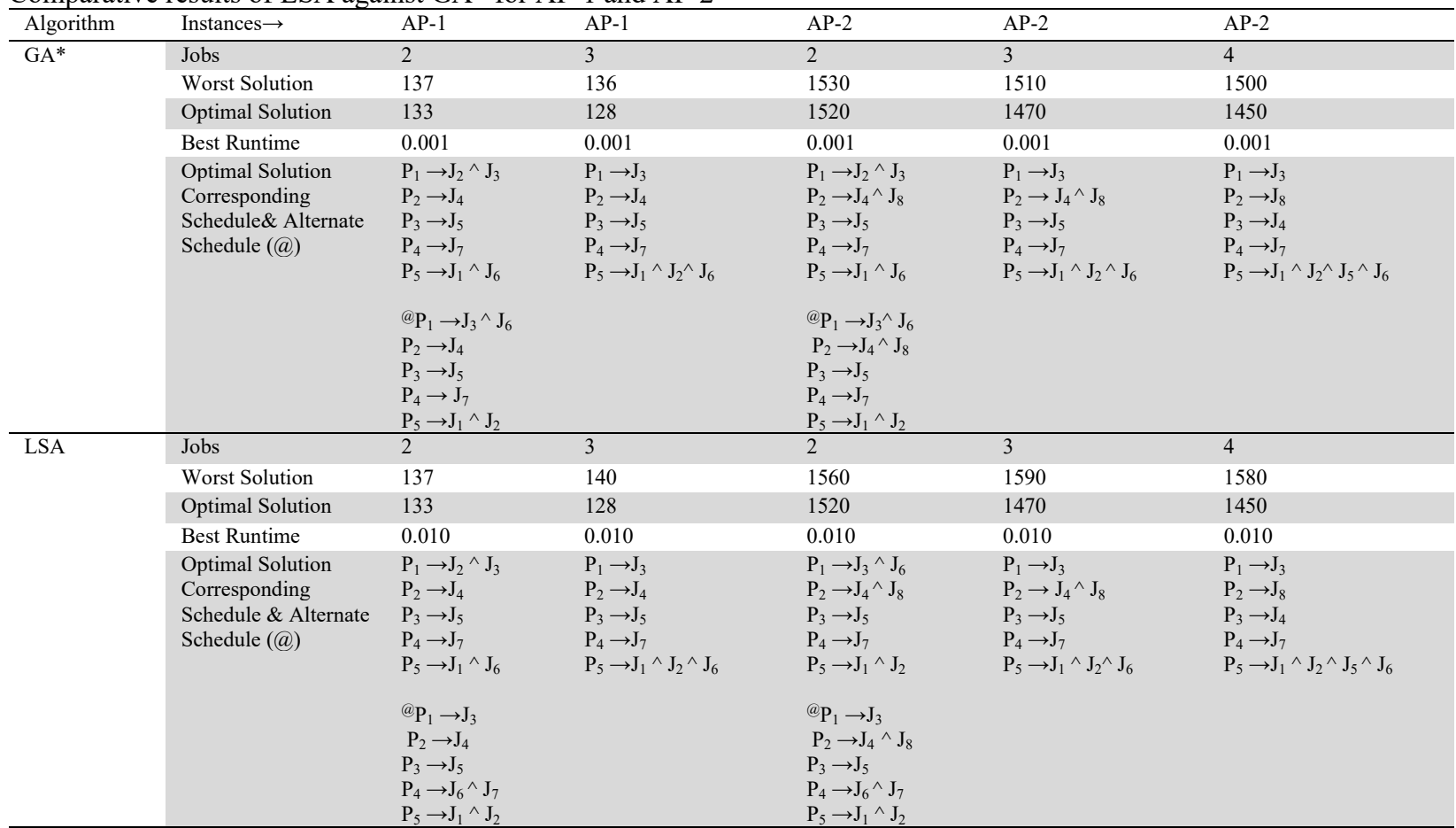

In Table 5, for all the instances of AP-1 and AP-2, the best results of LSA match with GA*. The CPU run time of LSA varies from 0.010 to 0.014 seconds, whereas GA* converges to 0.001 seconds. In Table 6 and Table 7, the best solutions acheived through LSA match with GA* for 4 cases, such as AP-3 (with 2 and 4 jobs) and AP-4 (with 2 and 3 jobs).

Table 6

Comparative results of LSA against GA* for AP-3

\begin{tabular}{|c|c|c|c|c|c|}
\hline Algorithm & Instances $\rightarrow$ & AP-3 & AP-3 & AP-3 & AP-3 \\
\hline \multirow[t]{10}{*}{$\mathrm{GA}^{*}$} & Jobs & 2 & 3 & 4 & 5 \\
\hline & Worst Solution & 80 & 75 & 70 & 78 \\
\hline & Optimal Solution & 66 & 67 & 66 & 74 \\
\hline & Best Runtime & 0.060 & 0.060 & 0.060 & 0.060 \\
\hline & Optimal Solution & $\mathrm{P}_{1} \rightarrow \mathrm{J}_{2} \wedge \mathrm{J}_{5}$ & $\mathrm{P}_{1} \rightarrow \mathrm{J}_{2} \wedge \mathrm{J}_{5}$ & $\mathrm{P}_{1} \rightarrow \mathrm{J}_{2}$ & $\mathrm{P}_{1} \rightarrow \mathrm{J}_{2}$ \\
\hline & Corresponding & $\mathrm{P}_{2} \rightarrow \mathrm{J}_{4} \wedge \mathrm{J}_{7}$ & $\mathrm{P}_{2} \rightarrow \mathrm{J}_{4} \wedge \mathrm{J}_{6} \wedge \mathrm{J}_{7}$ & $\mathrm{P}_{2} \rightarrow \mathrm{J}_{4} \wedge \mathrm{J}_{5} \wedge \mathrm{J}_{6} \wedge \mathrm{J}_{7}$ & $\mathrm{P}_{2} \rightarrow \mathrm{J}_{4} \wedge \mathrm{J}_{5} \wedge \mathrm{J}_{6} \wedge \mathrm{J}_{7} \wedge \mathrm{J}_{10}$ \\
\hline & Schedule\& Alternate & $\mathrm{P}_{3} \rightarrow \mathrm{J}_{1} \wedge \mathrm{J}_{3}$ & $\mathrm{P}_{3} \rightarrow \mathrm{J}_{1} \wedge \mathrm{J}_{3}$ & $\mathrm{P}_{3} \rightarrow \mathrm{J}_{1} \wedge \mathrm{J}_{3}$ & $\mathrm{P}_{3} \rightarrow \mathrm{J}_{1}$ \\
\hline & Schedule(@) & $\mathrm{P}_{4} \rightarrow \mathrm{J}_{6}$ & $\mathrm{P}_{4} \rightarrow \mathrm{J}_{10}$ & $\mathrm{P}_{4} \rightarrow \mathrm{J}_{10}$ & $\mathrm{P}_{4} \rightarrow \mathrm{J}_{3}$ \\
\hline & & $\mathrm{P}_{5} \rightarrow \mathrm{J}_{8}$ & $\mathrm{P}_{5} \rightarrow \mathrm{J}_{8}$ & $\mathrm{P}_{5} \rightarrow \mathrm{J}_{8}$ & $\mathrm{P}_{5} \rightarrow \mathrm{J}_{8}$ \\
\hline & & $\mathrm{P}_{6} \rightarrow \mathrm{J}_{9} \wedge \mathrm{J}_{10}$ & $\mathrm{P}_{6} \rightarrow \mathrm{J}_{9}$ & $\mathrm{P}_{6} \rightarrow \mathrm{J}_{9}$ & $\mathrm{P}_{6} \rightarrow \mathrm{J}_{9}$ \\
\hline \multirow[t]{11}{*}{ LSA } & Jobs & 2 & 3 & 4 & 5 \\
\hline & Worst Solution & 72 & 71 & 68 & 71 \\
\hline & Optimal Solution & 66 & 65 & 66 & 69 \\
\hline & Best Runtime & 0.010 & 0.010 & 0.010 & 0.012 \\
\hline & Optimal Solution & $\mathrm{P}_{1} \rightarrow \mathrm{J}_{2} \wedge \mathrm{J}_{5}$ & $\mathrm{P}_{1} \rightarrow \mathrm{J}_{2}$ & $\mathrm{P}_{1} \rightarrow \mathrm{J}_{2}$ & $\mathrm{P}_{1} \rightarrow \mathrm{J}_{2}$ \\
\hline & Corresponding & $\mathrm{P}_{2} \rightarrow \mathrm{J}_{4} \wedge \mathrm{J}_{7}$ & $\mathrm{P}_{2} \rightarrow \mathrm{J}_{4} \wedge \mathrm{J}_{5} \wedge \mathrm{J}_{7}$ & $\mathrm{P}_{2} \rightarrow \mathrm{J}_{4} \wedge \mathrm{J}_{5} \wedge \mathrm{J}_{6} \wedge \mathrm{J}_{7}$ & $\mathrm{P}_{2} \rightarrow \mathrm{J}_{3} \wedge \mathrm{J}_{4} \wedge \mathrm{J}_{5} \wedge \mathrm{J}_{6} \wedge \mathrm{J}_{7}$ \\
\hline & Schedule \& Alternate & $\mathrm{P}_{3} \rightarrow \mathrm{J}_{1} \wedge \mathrm{J}_{3}$ & $\mathrm{P}_{3} \rightarrow \mathrm{J}_{1} \wedge \mathrm{J}_{3}$ & $\mathrm{P}_{3} \rightarrow \mathrm{J}_{1} \wedge \mathrm{J}_{3}$ & $\mathrm{P}_{3} \rightarrow \mathrm{J}_{1}$ \\
\hline & Schedule(@) & $\mathrm{P}_{4} \rightarrow \mathrm{J}_{6}$ & $\mathrm{P}_{4} \rightarrow \mathrm{J}_{6}$ & $\mathrm{P}_{4} \rightarrow \mathrm{J}_{10}$ & $\mathrm{P}_{4} \rightarrow \mathrm{J}_{10}$ \\
\hline & & $\mathrm{P}_{5} \rightarrow \mathrm{J}_{8}$ & $\mathrm{P}_{5} \rightarrow \mathrm{J}_{8}$ & $\mathrm{P}_{5} \rightarrow \mathrm{J}_{8}$ & $\mathrm{P}_{5} \rightarrow \mathrm{J}_{8}$ \\
\hline & & $\mathrm{P}_{6} \rightarrow \mathrm{J}_{9} \wedge \mathrm{J}_{10}$ & $\mathrm{P}_{6} \rightarrow \mathrm{J}_{9} \wedge \mathrm{J}_{10}$ & $\mathrm{P}_{6} \rightarrow \mathrm{J}_{9}$ & $\mathrm{P}_{6} \rightarrow \mathrm{J}_{9}$ \\
\hline & & & & & $\begin{array}{l}\text { @ } \mathrm{P}_{1} \rightarrow \mathrm{J}_{2} \\
\mathrm{P}_{2} \rightarrow \mathrm{J}_{1} \wedge \mathrm{J}_{4} \wedge \mathrm{J}_{5} \wedge \mathrm{J}_{6} \wedge \mathrm{J}_{7} \\
\mathrm{P}_{3} \rightarrow \mathrm{J}_{3} \\
\mathrm{P}_{4} \rightarrow \mathrm{J}_{10} \\
\mathrm{P}_{5} \rightarrow \mathrm{J}_{8} \\
\mathrm{P}_{6} \rightarrow \mathrm{J}_{9}\end{array}$ \\
\hline
\end{tabular}

For remaining cases, specifically AP-3 (with 3 and 5 jobs) and AP-4 (with 4 jobs) LSA provided the preferable results than the $\mathrm{GA}^{*}$. The computational run time of LSA ranges from 0.010 to 0.015 seconds, whereas $\mathrm{GA}^{*}$ ranges from 0.060 to 0.120 seconds. Here, LSA seems more competent than $\mathrm{GA}^{*}$ as per with solution quality and runtime aspects. It is evident that the 
LSA is also list out the alternative solutions efficiently, if it occurs. The other best found solutions can also be seen in Tables 5-7. This indicates the ability of LSA to list out all the possible alternative solutions. The overall results show that the LSA is outperforming the $\mathrm{GA}^{*}$ in both quality of the solution and runtime of the algorithm aspects.

Table 7

Comparative results of LSA against GA* for AP-4

\begin{tabular}{|c|c|c|c|c|}
\hline Algorithm & Instances $\rightarrow$ & AP-4 & AP-4 & AP-4 \\
\hline \multirow{18}{*}{$\mathrm{GA}^{*}$} & Jobs & 2 & 3 & 4 \\
\hline & Worst Solution & 71 & 75 & 82 \\
\hline & Optimal Solution & 69 & 69 & 73 \\
\hline & Best Runtime & 0.120 & 0.100 & 0.110 \\
\hline & Optimal Solution & $\mathrm{P}_{1} \rightarrow \mathrm{J}_{4}$ & $\mathrm{P}_{1} \rightarrow \mathrm{J}_{4}$ & $\mathrm{P}_{1} \rightarrow \mathrm{J}_{4}$ \\
\hline & Corresponding & $\mathrm{P}_{2} \rightarrow \mathrm{J}_{6} \wedge \mathrm{J}_{7}$ & $\mathrm{P}_{2} \rightarrow \mathrm{J}_{6}$ & $\mathrm{P}_{2} \rightarrow \mathrm{J}_{10}$ \\
\hline & Schedule\& Alternate & $\mathrm{P}_{3} \rightarrow \mathrm{J}_{1}$ & $\mathrm{P}_{3} \rightarrow \mathrm{J}_{10}$ & $\mathrm{P}_{3} \rightarrow \mathrm{J}_{1}$ \\
\hline & Schedule(@) & $\mathrm{P}_{4} \rightarrow \mathrm{J}_{3} \wedge \mathrm{J}_{8}$ & $\mathrm{P}_{4} \rightarrow \mathrm{J}_{3} \wedge \mathrm{J}_{7} \wedge \mathrm{J}_{8}$ & $\mathrm{P}_{4} \rightarrow \mathrm{J}_{3} \wedge \mathrm{J}_{6} \wedge \mathrm{J}_{7} \wedge \mathrm{J}_{8}$ \\
\hline & & $\mathrm{P}_{5} \rightarrow \mathrm{J}_{9}$ & $\mathrm{P}_{5} \rightarrow \mathrm{J}_{9}$ & \\
\hline & & $\mathrm{P}_{6} \rightarrow \mathrm{J}_{5}$ & $\mathrm{P}_{6} \rightarrow \mathrm{J}_{1} \wedge \mathrm{J}_{5}$ & $\mathrm{P}_{6} \rightarrow \mathrm{J}_{5}$ \\
\hline & & $\mathrm{P}_{7} \rightarrow \mathrm{J}_{2} \wedge \mathrm{J}_{10}$ & $\mathrm{P}_{7} \rightarrow \mathrm{J}_{2}$ & $\mathrm{P}_{7} \rightarrow \mathrm{J}_{2}$ \\
\hline & & (a) $\mathrm{P}_{1} \rightarrow \mathrm{J}_{4}$ & (a) $\mathrm{P}_{1} \rightarrow \mathrm{J}_{4}$ & \\
\hline & & $\mathrm{P}_{2} \rightarrow \mathrm{J}_{6} \wedge \mathrm{J}_{7}$ & $\mathrm{P}_{2} \rightarrow \mathrm{J}_{7}$ & \\
\hline & & $\mathrm{P}_{3} \rightarrow \mathrm{J}_{10}$ & $\mathrm{P}_{3} \rightarrow \mathrm{J}_{10}$ & \\
\hline & & $\mathrm{P}_{4} \rightarrow \mathrm{J}_{3} \wedge \mathrm{J}_{8}$ & $\mathrm{P}_{4} \rightarrow \mathrm{J}_{3} \wedge \mathrm{J}_{6} \wedge \mathrm{J}_{8}$ & \\
\hline & & $\mathrm{P}_{5} \rightarrow \mathrm{J}_{9}$ & $\mathrm{P}_{5} \rightarrow \mathrm{J}_{9}$ & \\
\hline & & $\mathrm{P}_{6} \rightarrow \mathrm{J}_{1} \wedge \mathrm{J}_{5}$ & $\mathrm{P}_{6} \rightarrow \mathrm{J}_{1} \wedge \mathrm{J}_{5}$ & \\
\hline & & $\mathrm{P}_{7} \rightarrow \mathrm{J}_{2}$ & $\mathrm{P}_{7} \rightarrow \mathrm{J}_{2}$ & \\
\hline \multirow[t]{17}{*}{ LSA } & Jobs & 2 & 3 & 4 \\
\hline & Worst Solution & 69 & 69 & 83 \\
\hline & Optimal Solution & 69 & 69 & 71 \\
\hline & Best Runtime & 0.010 & 0.011 & 0.012 \\
\hline & Optimal Solution & $\mathrm{P}_{1} \rightarrow \mathrm{J}_{4}$ & $\mathrm{P}_{1} \rightarrow \mathrm{J}_{4}$ & $\mathrm{P}_{1} \rightarrow \mathrm{J}_{4}$ \\
\hline & Corresponding & $\mathrm{P}_{2} \rightarrow \mathrm{J}_{6}$ & $\mathrm{P}_{2} \rightarrow \mathrm{J}_{6}$ & $\mathrm{P}_{2} \rightarrow \mathrm{J}_{6}$ \\
\hline & Schedule \& Alternate & $\mathrm{P}_{3} \rightarrow \mathrm{J}_{7} \wedge \mathrm{J}_{10}$ & $\mathrm{P}_{3} \rightarrow \mathrm{J}_{10}$ & $\mathrm{P}_{3} \rightarrow \mathrm{J}_{10}$ \\
\hline & Schedule(@) & $\mathrm{P}_{4} \rightarrow \mathrm{J}_{3} \wedge \mathrm{J}_{8}$ & $\mathrm{P}_{4} \rightarrow \mathrm{J}_{3} \wedge \mathrm{J}_{7} \wedge \mathrm{J}_{8}$ & $\mathrm{P}_{4} \rightarrow \mathrm{J}_{3} \wedge \mathrm{J}_{5} \wedge \mathrm{J}_{7} \wedge \mathrm{J}_{8}$ \\
\hline & & $\mathrm{P}_{5} \rightarrow \mathrm{J}_{9}$ & $\mathrm{P}_{5} \rightarrow \mathrm{J}_{9}$ & $\mathrm{P}_{5} \rightarrow \mathrm{J}_{9}$ \\
\hline & & $\mathrm{P}_{6} \rightarrow \mathrm{J}_{1} \wedge \mathrm{J}_{5}$ & $\mathrm{P}_{6} \rightarrow \mathrm{J}_{1} \wedge \mathrm{J}_{5}$ & $\mathrm{P}_{6} \rightarrow \mathrm{J}_{1}$ \\
\hline & & $\mathrm{P}_{7} \rightarrow \mathrm{J}_{2}$ & $\mathrm{P}_{7} \rightarrow \mathrm{J}_{2}$ & $\mathrm{P}_{7} \rightarrow \mathrm{J}_{2}$ \\
\hline & & (a) $\mathrm{P}_{1} \rightarrow \mathrm{J}_{4}$ & (a) $\mathrm{P}_{1} \rightarrow \mathrm{J}_{4}$ & \\
\hline & & $\mathrm{P}_{2} \rightarrow \mathrm{J}_{6} \wedge \mathrm{J}_{7}$ & $\mathrm{P}_{2} \rightarrow \mathrm{J}_{7}$ & \\
\hline & & $\mathrm{P}_{3} \rightarrow \mathrm{J}_{10}$ & $\mathrm{P}_{3} \rightarrow \mathrm{J}_{10}$ & \\
\hline & & $\mathrm{P}_{4} \rightarrow \mathrm{J}_{3} \wedge \mathrm{J}_{8}$ & $\mathrm{P}_{4} \rightarrow \mathrm{J}_{3} \wedge \mathrm{J}_{6} \wedge \mathrm{J}_{8}$ & \\
\hline & & $\mathrm{P}_{5} \rightarrow \mathrm{J}_{9}$ & $\mathrm{P}_{5} \rightarrow \mathrm{J}_{9}$ & \\
\hline & & $\mathrm{P}_{6} \rightarrow \mathrm{J}_{1} \wedge \mathrm{J}_{5}$ & $\mathrm{P}_{6} \rightarrow \mathrm{J}_{1} \wedge \mathrm{J}_{5}$ & \\
\hline
\end{tabular}

\subsection{Computational results of $k$-UAP using proposed LSA}

This section reports the computational details of $k$-UAP tested using the proposed LSA. For computational experiments, we have considered the same four test instances as used by Majumdar and Bhunia (2012). Each of these four instances with distinct $k$ values were tested using the proposed LSA. Each test instance is executed in ten independent runs with distinct $T S$ values and the summary of best found results are reported in Table 8 . Table 8 consists of the results for each instance such as the worst, optimal solutions, CPU runtime (in seconds) required to get the optimal solution and schedule corresponding to the optimal solution.

From Table 8, the key remarks are observed:

i. $\quad$ The proposed LSA produces new best solutions that may help to the future comparative purposes.

ii. The proposed LSA is proficient in achieving the substitute solutions, if exists.

iii. It is note that the test instance AP- 2 with $k=2$ could take 0.031 seconds while AP-2 with $k=4$ has taken 0.043 seconds to get optimal solution. Similarly, AP-3 with $k=3$ took 0.042 seconds whereas the test instance AP-3 with with $k=5$ has used 0.028 seconds to get the optimal solution. This clearly demonstrations the performance of the LSA depends on $k$ values.

iv. The runtime ranging from 0.027 seconds to 0.050 seconds show that the LSA takes less time for getting best solutions.

To conclude, the LSA appears to be more capable in providing promising solutions within considerably less time. 
Table 8

Computational results of $k-U A P$ using LSA on AP-1, AP-2, AP-3 \& AP-4

\begin{tabular}{|c|c|c|c|c|c|}
\hline Algorithm & Instances $\rightarrow$ & AP-1 & AP-1 & AP-1 & AP-2 \\
\hline \multirow{23}{*}{ LSA } & $k$ & 2 & 3 & 4 & 2 \\
\hline & Worst solution & 122 & 129 & 139 & 1400 \\
\hline & Optimal solution & 122 & 123 & 125 & 1400 \\
\hline & $\begin{array}{l}\text { Best runtime } \\
\text { (in Sec.) }\end{array}$ & 0.029 & 0.038 & 0.044 & 0.031 \\
\hline & $\begin{array}{l}\text { Optimal solution } \\
\text { corresponding } \\
\text { schedule \& alternate } \\
\text { schedule(@) }\end{array}$ & $\begin{array}{l}\mathrm{P}_{1} \rightarrow \mathrm{J}_{3} \\
\mathrm{P}_{5} \rightarrow \mathrm{J}_{1} \wedge \mathrm{J}_{2} \wedge \quad \mathrm{J}_{4} \wedge \\
\mathrm{J}_{5} \wedge \mathrm{J}_{6} \wedge \mathrm{J}_{7}\end{array}$ & $\begin{array}{l}\mathrm{P}_{1} \rightarrow \mathrm{J}_{3} \\
\mathrm{P}_{2} \rightarrow \mathrm{J}_{4} \\
\mathrm{P}_{5} \rightarrow \mathrm{J}_{1} \wedge \mathrm{J}_{2} \wedge \mathrm{J}_{5} \wedge \\
\mathrm{J}_{6} \wedge \mathrm{J}_{7}\end{array}$ & $\begin{array}{l}\mathrm{P}_{1} \rightarrow \mathrm{J}_{3} \\
\mathrm{P}_{2} \rightarrow \mathrm{J}_{4} \\
\mathrm{P}_{4} \rightarrow \mathrm{J}_{7} \\
\mathrm{P}_{5} \rightarrow \mathrm{J}_{1} \wedge \mathrm{J}_{2} \wedge \mathrm{J}_{5} \wedge \mathrm{J}_{6}\end{array}$ & $\begin{array}{l}P_{1} \rightarrow J_{3} \\
P_{5} \rightarrow J_{1} \wedge J_{2} \wedge J_{4} \wedge J_{5} \wedge J_{6} \wedge J_{7}\end{array}$ \\
\hline & Instances $\rightarrow$ & AP-2 & AP-2 & AP-3 & AP-3 \\
\hline & $k$ & 3 & 4 & 2 & 3 \\
\hline & Worst solution & 1470 & 1570 & 86 & 78 \\
\hline & Optimal solution & 1410 & 1430 & 76 & 68 \\
\hline & $\begin{array}{l}\text { Best runtime } \\
\text { (in Sec.) }\end{array}$ & 0.028 & 0.043 & 0.042 & 0.042 \\
\hline & $\begin{array}{l}\text { Optimal solution } \\
\text { corresponding } \\
\text { schedule \& alternate } \\
\text { schedule(@) }\end{array}$ & $\begin{array}{l}P_{1} \rightarrow J_{3} \\
P_{2} \rightarrow J_{8} \\
P_{5} \rightarrow J_{1} \wedge J_{2} \wedge J_{4} \wedge J_{5} \wedge \\
J_{6} \wedge J_{7}\end{array}$ & $\begin{array}{l}\mathrm{P}_{1} \rightarrow \mathrm{J}_{3} \\
\mathrm{P}_{2} \rightarrow \mathrm{J}_{8} \\
\mathrm{P}_{3} \rightarrow \mathrm{J}_{4} \\
\mathrm{P}_{5} \rightarrow \mathrm{J}_{1} \wedge \mathrm{J}_{2} \wedge \mathrm{J}_{5} \wedge \\
\mathrm{J}_{6} \wedge \mathrm{J}_{7}\end{array}$ & $\begin{array}{l}\mathrm{P}_{2} \rightarrow \mathrm{J}_{1} \wedge \mathrm{J}_{3} \wedge \mathrm{J}_{4} \wedge \mathrm{J}_{5} \wedge \mathrm{J}_{6} \wedge \\
\mathrm{J}_{7} \wedge \mathrm{J}_{8} \wedge \mathrm{J}_{10} \\
\mathrm{P}_{6} \rightarrow \mathrm{J}_{2} \wedge \mathrm{J}_{9}\end{array}$ & $\begin{array}{l}\mathrm{P}_{2} \rightarrow \mathrm{J}_{4} \wedge \mathrm{J}_{5} \wedge \mathrm{J}_{6} \wedge \mathrm{J}_{7} \\
\mathrm{P}_{3} \rightarrow \mathrm{J}_{1} \wedge \mathrm{J}_{3} \wedge \mathrm{J}_{8} \\
\mathrm{P}_{5} \rightarrow \mathrm{J}_{2} \wedge \mathrm{J}_{9} \wedge \mathrm{J}_{10}\end{array}$ \\
\hline & Instances $\rightarrow$ & $\mathrm{AP}-3$ & $\mathrm{AP}-3$ & $\mathrm{AP}-4$ & AP-4 \\
\hline & $k$ & 4 & 5 & 2 & 3 \\
\hline & Worst solution & 63 & 61 & 103 & 83 \\
\hline & Optimal solution & 63 & 61 & 90 & 73 \\
\hline & $\begin{array}{l}\text { Best runtime } \\
\text { (in Sec.) }\end{array}$ & 0.030 & 0.027 & 0.050 & 0.039 \\
\hline & $\begin{array}{l}\text { Optimal solution } \\
\text { corresponding } \\
\text { schedule \& alternate } \\
\text { schedule(@) }\end{array}$ & $\begin{array}{l}\mathrm{P}_{1} \rightarrow \mathrm{J}_{2} \\
\mathrm{P}_{2} \rightarrow \mathrm{J}_{4} \wedge \mathrm{J}_{5} \wedge \mathrm{J}_{6} \wedge \mathrm{J}_{7} \\
\mathrm{P}_{3} \rightarrow \mathrm{J}_{1} \wedge \mathrm{J}_{3} \wedge \mathrm{J}_{8} \\
\mathrm{P}_{6} \rightarrow \mathrm{J}_{9} \wedge \mathrm{J}_{10}\end{array}$ & $\begin{array}{l}\mathrm{P}_{1} \rightarrow \mathrm{J}_{2} \\
\mathrm{P}_{2} \rightarrow \mathrm{J}_{4} \wedge \mathrm{J}_{5} \wedge \mathrm{J}_{6} \wedge \\
\mathrm{J}_{7} \\
\mathrm{P}_{3} \rightarrow \mathrm{J}_{1} \wedge \mathrm{J}_{3} \\
\mathrm{P}_{5} \rightarrow \mathrm{J}_{8} \\
\mathrm{P}_{6} \rightarrow \mathrm{J}_{9} \wedge \mathrm{J}_{10}\end{array}$ & $\begin{array}{l}\mathrm{P}_{4} \rightarrow \mathrm{J}_{1} \wedge \mathrm{J}_{3} \wedge \mathrm{J}_{5} \wedge \mathrm{J}_{6} \wedge \mathrm{J}_{7} \wedge \\
\mathrm{J}_{8} \\
\mathrm{P}_{7} \rightarrow \mathrm{J}_{2} \wedge \mathrm{J}_{4} \wedge \mathrm{J}_{9} \wedge \mathrm{J}_{10}\end{array}$ & $\begin{array}{l}\mathrm{P}_{4} \rightarrow \mathrm{J}_{3} \wedge \mathrm{J}_{6} \wedge \mathrm{J}_{7} \wedge \mathrm{J}_{8} \\
\mathrm{P}_{5} \rightarrow \mathrm{J}_{1} \wedge \mathrm{J}_{5} \wedge \mathrm{J}_{9} \\
\mathrm{P}_{7} \rightarrow \mathrm{J}_{2} \wedge \mathrm{J}_{4} \wedge \mathrm{J}_{10}\end{array}$ \\
\hline & Instances $\rightarrow$ & AP-4 & $\mathrm{AP}-4$ & AP-4 & \\
\hline & $k$ & 4 & 5 & 6 & \\
\hline & Worst solution & 78 & 73 & 68 & \\
\hline & Optimal solution & 68 & 68 & 68 & \\
\hline & $\begin{array}{l}\text { Best runtime } \\
\text { (in Sec.) }\end{array}$ & 0.035 & 0.036 & 0.033 & \\
\hline & $\begin{array}{l}\text { Optimal solution } \\
\text { corresponding } \\
\text { schedule \& alternate } \\
\text { schedule(@) }\end{array}$ & $\begin{array}{l}\mathrm{P}_{4} \rightarrow \mathrm{J}_{3} \wedge \mathrm{J}_{6} \wedge \mathrm{J}_{7} \wedge \mathrm{J}_{8} \\
\mathrm{P}_{5} \rightarrow \mathrm{J}_{9} \\
\mathrm{P}_{6} \rightarrow \mathrm{J}_{1} \wedge \mathrm{J}_{5} \\
\mathrm{P}_{7} \rightarrow \mathrm{J}_{2} \wedge \mathrm{J}_{4} \wedge \mathrm{J}_{10}\end{array}$ & $\begin{array}{l}\mathrm{P}_{2} \rightarrow \mathrm{J}_{6} \wedge \mathrm{J}_{7} \\
\mathrm{P}_{4} \rightarrow \mathrm{J}_{3} \wedge \mathrm{J}_{8} \\
\mathrm{P}_{5} \rightarrow \mathrm{J}_{9} \\
\mathrm{P}_{6} \rightarrow \mathrm{J}_{1} \wedge \mathrm{J}_{5} \\
\mathrm{P}_{7} \rightarrow \mathrm{J}_{2} \wedge \mathrm{J}_{4} \wedge \mathrm{J}_{10}\end{array}$ & $\begin{array}{l}\mathrm{P}_{1} \rightarrow \mathrm{J}_{4} \\
\mathrm{P}_{2} \rightarrow \mathrm{J}_{6} \wedge \mathrm{J}_{7} \\
\mathrm{P}_{4} \rightarrow \mathrm{J}_{3} \wedge \mathrm{J}_{8} \\
\mathrm{P}_{5} \rightarrow \mathrm{J}_{9} \\
\mathrm{P}_{6} \rightarrow \mathrm{J}_{1} \wedge \mathrm{J}_{5} \\
\mathrm{P}_{7} \rightarrow \mathrm{J}_{2} \wedge \mathrm{J}_{10}\end{array}$ & \\
\hline
\end{tabular}

\section{Conclusion}

Due to the inadequacy of the workforce, various assignment models can be seen as problems in which the number of persons is fewer than the number of jobs (i.e. $m<n)$ ). Thus, it is inevitable to assign multiple jobs to a single person in an effective manner. To address one such scenario, the more generalized variant of UAP called " $k$-cardinality unbalanced assignment problem" $(k-U A P)$ is considered in this study. In addition, this model also includes an additional constraint over the total jobs that a person is permitted to do and a cardinality over the set of persons. This model has been formulated using 0-1 ILP. An exact LSA is developed to solve $k$-UAP optimally. Comparative results showed that the proposed LSA is competent over existing algorithm. Computational results of four test instances with distinct cardinalities have been provided. The overall results showed that the proposed LSA may be competent over the existing algorithms in providing best results of $k$-UAP.

\section{References}

Arora, S., \& Puri, M. C. (1998). A variant of time minimizing assignment problem. European Journal of Operational Research, 110(2), 314-325.

Bai, G. Z. (2009, December). A new algorithm for k-cardinality assignment problem. In 2009 International Conference on Computational Intelligence and Software Engineering (pp. 1-4). IEEE. 
Belik, I., \& Jörnsten, K. (2016). A new Semi-Lagrangean Relaxation for the k-cardinality assignment problem. Journal of Information and Optimization Sciences, 37(1), 75-100.

Bhavani, V., \& Murthy, M. S. (2006). Truncated M-travelling salesmen problem. Opsearch, 43(2), 152-177.

Bhunia, A. K., Biswas, A., \& Samanta, S. S. (2017). A genetic algorithm-based approach for unbalanced assignment problem in interval environment. International Journal of Logistics Systems and Management, 27(1), 62-77.

Dell'Amico, M., \& Martello, S. (1997). The k-cardinality assignment problem. Discrete Applied Mathematics, 76(1-3), 103121.

Dell'Amico, M., Lodi, A., \& Martello, S. (2001). Efficient algorithms and codes for k-cardinality assignment problems. Discrete Applied Mathematics, 110(1), 25-40.

Feng, Y., \& Yang, L. (2006). A two-objective fuzzy k-cardinality assignment problem. Journal of Computational and Applied Mathematics, 197(1), 233-244.

Gabrovšek, B., Novak, T., Povh, J., Rupnik Poklukar, D., \& Žerovnik, J. (2020). Multiple Hungarian Method for kAssignment Problem. Mathematics, 8(11), 2050.

Iampang, A., Boonjing, V., \& Chanvarasuth, P. (2010, December). A cost and space efficient method for unbalanced assignment problems. In 2010 IEEE International Conference on Industrial Engineering and Engineering Management (pp. 985-988). IEEE.

Votaw, D.F., \& Orden, A. (1952) 'The personnel assignment problem', Symposium on Linear Inequalities and Programming, Scoop 10, US Air Force, pp.155-163.

Kuhn, H. W. (1955). The Hungarian method for the assignment problem. Naval Research Logistics Quarterly, 2(1-2), 83-97.

Kumar, A. (2006). A modified method for solving the unbalanced assignment problems. Applied Mathematics and Computation, 176(1), 76-82.

Kumar, T. J., \& Purusotham, S. (2017). An exact algorithm for k-cardinality degree constrained clustered minimum spanning tree problem. In IOP Conf. Ser. Mater. Sci. Eng. (Vol. 263, p. 042112).

Kumar, T., \& Purusotham, S. (2018). The degree constrained k-cardinality minimum spanning tree problem: a lexi-search algorithm. Decision Science Letters, 7(3), 301-310.

Majumdar, J., \& Bhunia, A. K. (2012). An alternative approach for unbalanced assignment problem via genetic algorithm. Applied Mathematics and Computation, 218(12), 6934-6941.

Malhotra, R., \& Bhatia, H. L. (1984). Variants of the time minimization assignment problem. Trab. Estad. Invest. Oper., 35(3), 331-338.

Sundara Murthy, M. (1976). A bulk transportation problem. Opsearch, 13(3-4), 143-155.

Thenepalle, J. K., \& Singamsetty, P. (2018). Bi-criteria travelling salesman subtour problem with time threshold. The European Physical Journal Plus, 133(3), 1-15.

Thenepalle, J. K., \& Singamsetty, P. (2019). Lexi-search algorithm for one to many multidimensional bi-criteria unbalanced assignment problem. International Journal of Bio-Inspired Computation, 14(3), 151-170.

Volgenant, A. (2004). Solving the k-cardinality assignment problem by transformation. European Journal of Operational Research, 157(2), 322-331.

Yadaiah, V., \& Haragopal, V. V. (2016). A new approach of solving single objective unbalanced assignment problem. American Journal of Operations Research, 6(1), 81-89.

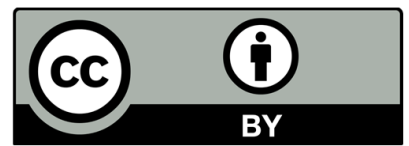

(C) 2022 by the authors; licensee Growing Science, Canada. This is an open access article distributed under the terms and conditions of the Creative Commons Attribution (CCBY) license (http://creativecommons.org/licenses/by/4.0/). 